01

\title{
Вейвлет корреляции нестационарных сигналов
}

\author{
(C) С.В. Божокин, С.В. Жарко, Н.В. Ларионов, А.Н. Литвинов, И.М. Соколов \\ Санкт-Петербургский политехнический университет Петра Великого, \\ 195251 Санкт-Петербург, Россия \\ e-mail: bsvjob@mail.ru
}

(Поступило в Редакцию 29 декабря 2015 г. В окончательной редакции 20 сентября 2016 г.)

\begin{abstract}
Предложены и проанализированы два новых подхода к анализу нестационарных случайных сигналов. Первый подход основан на введении адаптивного вейвлета Морле, позволяющего изменять временное и частотное разрешения исследуемых сигналов с помощью вспомогательного управляющего параметра. Второй подход связан с использованием новой двойной корреляционной функции, которая представляет собой корреляцию непрерывных вейвлетных преобразований двух сигналов, вычисленных как по частоте, так и по времени. Отмечены преимущества введенной корреляционной функции перед другими корреляционными функциями, в частности, возможности анализировать не только временные, но и частотные корреляции нестационарных сигналов. Обсуждены применения разработанных подходов для анализа различных переходных процессов в физике.
\end{abstract}

DOI: $10.21883 / J T F .2017 .06 .44502 .1721$

\section{Введение}

Различные сигналы в физике $Z(t)$, зависящие от времени $t$, можно разделить на две группы: стационарные и нестационарные. Стационарные случайные процессы обладают тем свойством, что их статистические и спектральные характеристики, определенные для достаточно большого интервала времени $T$, не изменяются, если взять любой другой интервал времени $T$, смещенный относительно первого на произвольный отрезок времени. Для стационарного случайного процесса среднее значение $\overline{z(t)}$, среднеквадратичное значение $\overline{z^{2}(t)}$ не зависят от времени $t$, причем усреднение производится по большому числу реализаций $k$ случайного процесса. Автокорреляционная функция стационарного случайного процесса $\overline{\mathrm{CF}_{z z}(t, t+\tau)}$ также не зависит от времени $t$, а зависит только от времени $\tau$ - разности двух моментов времени $t+\tau$ и $t$, в которых производится усреднение.

Для описания стационарных сигналов часто используются преобразования Фурье [1-4]. В этом случае для двух сигналов $Z_{\alpha}(t)$ и $Z_{\beta}(t)$, имеющих фурье-компоненты $Z_{\alpha}(v)$ и $Z_{\beta}(v)$, где $v$ - частота, можно ввести кросскорреляционную функцию $\mathrm{CCF}_{\alpha \beta}(t)[4,5]$. Для анализа изменения спектральных свойств сигнала по времени весь интервал измерения сигнала $T$ обычно делится на $M$ эпох равной продолжительности. Для каждой эпохи, которые могут перекрывать друг друга, выполняется оконное преобразования Фурье (STFT - Short Time Fourier Transform), причем размер окна $W_{0}$ равняется продолжительности эпохи. Выбор оптимального размера окна $W_{0}$ представляет собой самостоятельную задачу. Окно большой продолжительности позволяет получить хорошее разрешение по частоте, но плохое по времени. Слишком широкое окно будет полезно для обнаружения низкочастотных компонент сигнала, но его ширина будет избыточной для обнаружения быстроизменяющихся гармоник с большой частотой. Таким образом, выбор оптимального размера эпохи $W_{0}$ требует знания характерных масштабов времени, за которые происходят перестройки спектральных свойств исследуемого сигнала.

Производя процедуру усреднения Фурье компонент исследуемых сигналов по различным эпохам $\left\langle Z_{\alpha}(v) Z_{\beta}^{*}(v)\right\rangle[6,7]$, можно ввести квадрат когерентности (coherence) двух сигналов $\gamma_{\alpha \beta}^{2}(v)$. К сожалению, квадрат когерентности зависит от процедуры усреднения, от выбора величины окна, от вида окна, от величины сдвига шага окна, поэтому величина $\gamma_{\alpha \beta}^{2}(v)$ не может являться строгой количественной мерой скоррелированности двух сигналов $Z_{\alpha}(t)$ и $Z_{\beta}(t)$ [8-11].

Для нестационарных процессов (NS) различные функции распределения изменяются при сдвиге по оси времени $t$ [4]. Это означает, что функции распределения NS, а также амплитудно-частотные характеристики NS меняются с течением времени. Для исследования NS успешно применяется непрерывное вейвлетное преобразование (CWT) [1-3,12-14]. Сравнение метода CWT с другими методами изучения NS выполнено в работах [7,15-17].

Предположим, что сигнал $Z_{\alpha}(t)$ имеет CWT, равное $V_{\alpha}(v, t)$, а сигнал $Z_{\beta}(t)$ имеет CWT, равное $V_{\beta}(v, t)$. В этом случае можно определить вейвлетный взаимный спектр (Cross Wavelet Spectrum) $\operatorname{CWS}_{\alpha \beta}(v, t)=$ $=V_{\alpha}(v, t) V_{\beta}^{*}(v, t)$. Усредняя функцию $\operatorname{CWS}_{\alpha \beta}(v, t)$ как по частотам $v$, так и по интервалам времени $t$, и нормируя эту величину на единицу, можно получить квадрат вейвлетной функции когерентности (WCF Wavelet Coherence Function) $\Gamma^{2}(v, t)$. Детали процедуры усреднения функции $\mathrm{CWS}_{\alpha \beta}(v, t)$ для вычисления функции $\Gamma^{2}(v, t)$, а также ее применение к различным нестационарным сигналам можно найти в работах [6,18-23]. К сожалению, значения функции $\Gamma^{2}(v, t)$ зависят от типа материнского вейвлета, который используется для вычисления CWT, а также от процедуры усреднения по 
масштабам $v$ и $t$. Отметим, что процедура усреднения по времени WCF, связанная с идеей STFT, противоречит основной идее CWT изучения NS, спектральные и временные свойства которых могут значительно изменяться. Корреляции для таких сигналов NS могут развиваться и исчезать на малых масштабах по времени. Однако этот эффект функция $\Gamma^{2}(v, t)$, основанная на процедуре усреднения по многим эпохам, не может обнаружить. Корреляционные функции, построенные с помощью CWT, рассмотрены в работах $[16,24]$. В работе [16] рассматривается мгновенная корреляция двух различных сигналов $Z_{\alpha}(t)$ и $Z_{\beta}(t)$, основанная на вычислении спектральных интегралов CWT. В работе [24] вводится корреляция по времени $t$ двух CWT $V_{\alpha}(v, t)$ и $V_{\beta}(v, t)$.

В отличие от стандартного подхода CWT [1-3,15,23] в нашей работе используется новый адаптивный вейвлет Морле (AWM), который позволит улучшить спектральные разрешения NS при вычислении их корреляций. $\mathrm{B}$ настоящей работе будет предложена двойная вейвлетная корреляционная функция $\mathrm{DWC}_{\alpha \beta}(v, t)$, которая представляет собой корреляцию CWT двух нестационарных сигналов $Z_{\alpha}(t)$ и $Z_{\beta}(t)$, выполненную как по времени $t$, так и по частоте $v$. Для решения этих задач будет:

1) введен адаптивный материнский вейвлет Морле $\psi(x)$, позволяющий изменять как частотное, так и временное разрешения исследуемого NS с помощью изменения своего управляющего параметра $m$;

2) разработана математическая модель NS и получены аналитические выражения для CWT таких сигналов. В качестве модели NS будет рассмотрена суперпозиция элементарных нестационарных сигналов (ENS), представляющих собой произведение огибающей гауссовой формы на осциллирующую функцию с фиксированной частотой;

$3)$ предложена двойная $\operatorname{DWC}_{\alpha \beta}(v, t)$, которая позволит определить как разность двух частот исследуемых ENS, так и разность времен их локализации. Будет проведено сравнение введенной авторами настоящей работы функции $\mathrm{DWC}_{\alpha \beta}(v, t)$ с обычной кросскорреляционной функцией $\mathrm{CCF}_{\alpha \beta}(t)$.

Изучение суперпозиции ENS с помощью AWM, предлагаемое в настоящей работе, допускает аналитическое выражение для CWT. Это позволяет тестировать многие численные алгоритмы для реальных NS сигналов большой длительности. Предложенная в настоящей работе модель реального сигнала, представляющая собой суперпозицию двух ENS и случайного сигнала, продемонстрировала возможность улучшения спектрального разрешения CWT с помощью изменения управляющего параметра AWM. Разрабатываемая модель изучения корреляций $\mathrm{NS}$, использующая $\mathrm{DWC}_{\alpha \beta}(v, t)$, может быть использована: при изучении генерации нейронных спайков $[25,26]$, при вычислении динамики корреляций электроэнцефалограммы (EEG) различных отведений головного мозга $[27,28]$, при анализе циркадных ритмов животных [29], для характеристики нестабильности квантовых стандартов частоты [30,31], при анализе вспышечных процессов в астрофизике [32].

\section{Адаптивный вейвлет Морле}

Материнский вейвлет $\psi(x)$ должен быть хорошо локализован вблизи точки $x=0$, иметь нулевое среднее значение, вычисленное по всему интервалу переменной $-\infty<x<\infty$, и обладать единичной нормой (1) [1-3,33]. Этим свойствам удовлетворяет введенный нами AWM. Для оригинала AWM $\psi(x)$ и его фурьекомпоненты $\hat{\psi}(F)$ справедливы выражения

$$
\begin{gathered}
\psi(x)=D_{m} \exp \left(-\frac{x^{2}}{2 m^{2}}\right)\left[\exp (2 \pi i x)-\exp \left(-\Omega_{m}^{2}\right)\right] \\
\hat{\psi}(F)=\frac{D_{m} \Omega_{m}}{\sqrt{\pi} \exp \left[-\Omega_{m}^{2}(F-1)^{2}\right]\left[1-\exp \left(-2 \Omega_{m}^{2} F\right)\right]} \\
D_{m}=\frac{(2 \pi)^{1 / 4}}{\sqrt{\Omega_{m}\left(1-2 \exp \left(-\frac{3 \Omega_{m}^{2}}{2}\right)+\exp \left(-2 \Omega_{m}^{2}\right)\right)}}
\end{gathered}
$$

В выражениях (1), (3) величина $m$ играет роль управляющего параметра AWM, а величина $\Omega_{m}=m \pi \sqrt{2}$. Если мы рассмотрим отношение фурье-компонент $\hat{\psi}(F)$ на отрицательных и на положительных частотах, то это отношение равно $\hat{\psi}(-|F|) / \hat{\psi}(|F|)=-\exp \left[-2 \Omega_{m}^{2}|F|\right]$, причем на характерных частотах $F \approx 1$ этой величиной можно пренебречь. С большой точностью параметр локализации $\Delta_{x}[1,2]$, определяющий протяженность функции $\psi(x)$ по оси $x$, и параметр $\Delta_{F}$, показывающий протяженность частотного спектра функции $\hat{\psi}(F)[1,2]$, имеют значения $\Delta_{x} \approx m / \sqrt{2}, \Delta_{F} \approx 1 /(\sqrt{8} \pi m)$, а их произведение близко к наименьшему значению $\Delta_{x} \Delta_{F}=1 /(4 \pi)[1,2]$. Следовательно, в зависимости от параметра $m$ величины $\Delta_{x}$ и $\Delta_{F}$ AWM могут изменяться. Таким образом, при изменении $m$ появляется возможность изменения временного и частотного разрешения исследуемых NS. Если параметр $m=1$, то AWM (1) переходит в известное выражение для обычного вейвлета Морле [1-3], [23], [33,34]. Условие конечности константы $C_{\psi}$, определенной соотношением

$$
C_{\psi}=\int_{-\infty}^{\infty} \frac{|\hat{\psi}(F)|^{2} d F}{|F|},
$$

позволяет восстанавливать сигнал $Z(t)$ по его CWT $V(v, t)[1,2]$. Используя метод, позволяющий вычислять поправки к методу перевала [35], можно показать, что в случае $\Omega_{m} \gg 1$ асимптотическое выражение $C_{\psi} \approx 1+1 /\left(4 \Omega_{m}^{2}\right)$ отличается от точного численного значения $C_{\psi}$ менее, чем на $0.05 \%$ для значения $m>1$. Для материнских вейвлетов AWM, аргументы которых $x$ представляют собой комбинацию частот $v$, измеряемых 
в $\mathrm{Hz}$, и времен $t, t^{\prime}, t_{0}$, измеряемых в секундах, справедливо соотношение [36]

$$
\begin{gathered}
\frac{2}{C_{\psi}} \int_{0}^{\infty} d v v \int_{-\infty}^{\infty} d t_{0} \operatorname{Re}\left\{\psi^{*}\left[v\left(t^{\prime}-t_{0}\right)\right] \psi\left[v\left(t-t_{0}\right)\right]\right\} \\
=\delta\left(t-t^{\prime}\right)
\end{gathered}
$$

где $\delta\left(t-t^{\prime}\right)$ - дельта функция Дирака, а символ $\mathrm{Re}$ означает реальную часть выражения.

\section{Непрерывное вейвлетное преобразование}

Непрерывное вейвлет преобразование $V(v, t)$ (CWT) отображает нестационарный сигнал $Z(t)$ с изменяющейся частотно-временной структурой на плоскость времени $t$ и частоты $v[16,33,37-39]$

$$
V(v, t)=v \int_{-\infty}^{\infty} Z\left(t^{\prime}\right) \psi^{*}\left(v\left(t^{\prime}-t\right)\right) d t^{\prime}
$$

В этом выражении $\psi(x)$ - материнский вейвлет, а значок * означает комплексное сопряжение. Величина частоты $v>0$ определяет масштаб сжатия или растяжения материнского вейвлета. Аргумент $t$ определяет положение центра локализации вейвлета на оси времени. Преимущества введенного CWT $V(v, t)(6)$ разобраны в работе [16]. Для бесконечного гармонического сигнала $z(t)=\cos \left(2 \pi f_{0} t\right)$ с частотой $f_{0}$ максимум величины $|V(v, t)|$ (6) будет наблюдаться точно при условии $v=f_{0}$. Для двух бесконечных гармонических сигналов с одинаковыми амплитудами и частотами $f_{1}$ и $f_{2}$ максимумы хребтов с одинаковой амплитудой $V(v, t)(6)$ будут расположены в точках $v=f_{1}$ и $v=f_{2}$. Если характерная протяженность $\operatorname{AWM} \psi(x)$ равна $\Delta_{x} \approx m / \sqrt{2}$, то характерные моменты времени, вносящие основной вклад в интеграл (12), удовлетворяют соотношению

$$
t-\Delta_{x} / v<t^{\prime}<t+\Delta_{x} / v
$$

Адаптивный материнский вейвлет AWM (1), (2) играет роль изменяющегося окна, зависящего от управляющего параметра $m$, ширина которого становится большой для малых частот $v$ и малой для больших. Для нахождения CWT (6) бывает удобным использовать разложение всех величин в формуле (6) в интеграл Фурье

$$
V(v, t)=\int_{-\infty}^{\infty} \hat{Z}(f) \hat{\psi}^{*}\left(\frac{f}{v}\right) \exp (2 \pi i f t) d f
$$

где $\hat{Z}(f), \hat{\psi}^{*}(f / v)$ - фурье-компоненты сигнала $Z(t)$ и материнского вейвлета $\psi(x)(2)$. Для CWT справедливо соотношение, аналогичное формуле Парсеваля в фурьеанализе

$$
\int_{-\infty}^{\infty} z^{2}(t) d t=\frac{2}{C_{\psi}} \int_{-\infty}^{\infty} d t \int_{0}^{\infty} d v \frac{|V(v, t)|^{2}}{v} .
$$

Из формулы (9) видно, что величина $\varepsilon(v, t)$, определяемая соотношением

$$
\varepsilon(v, t)=\frac{2}{C_{\psi}} \frac{|V(v, t)|^{2}}{v},
$$

характеризует мгновенное распределение энергии сигнала по частотам $v$, вычисленное в момент времени $t$. Величина $\varepsilon(v, t)$, вычисленная с помощью CWT $V(v, t)(6)$, представляет собой локальную плотность спектра энергии сигнала, характеризующую спектральные свойства сигнала при заданной частоте $v$ и времени $t$.

Для многих сигналов важны изменения их спектральных свойств в некотором заданном интервале частот $\left[v_{L}, v_{R}\right]$. Вместо левой $v_{L}$ и правой $v_{R}$ границ диапазона удобно ввести среднюю частоту диапазона $v_{\mu}=\left(v_{L}+v_{R}\right) / 2$ и его ширину $\Delta v=v_{R}-v_{L}$. Для исследования динамики нарастания и спадания различных частот нестационарных сигналов вводится в рассмотрение спектральный интеграл $E_{\mu}(t)$ [37-39]

$$
E_{\mu}(t)=\frac{1}{\Delta v} \int_{v_{\mu}-\Delta v / 2}^{v_{\mu}+\Delta v / 2} \varepsilon(v, t) d v
$$

Спектральный интеграл $E_{\mu}(t)$ представляет собой среднее значение локальной плотности спектра энергии сигнала, проинтегрированное по рассматриваемому интервалу частот. Изучая поведение спектральных интегралов по времени $E_{\mu}(t)$, мы выполняем своеобразную фильтрацию нашего сигнала, суммируя вклады от локальной плотности спектра $\varepsilon(v, t)$ в определенном интервале частот $\mu$. Спектральные интегралы (11) были использованы в работе [16] для нахождения количественных характеристик возникновения и исчезновения корреляционных свойств различных NS.

Применение AWM позволит получить лучшие результаты для спектрального и временного разрешений $Z(t)$ по сравнению с использованием традиционного вейвлета Морле. Это легко увидеть уже для случая самых простых сигналов. Для бесконечного гармонического сигнала $Z(t)=\cos \left(2 \pi f_{0} t\right)$ с частотой $f_{0}$ можно получить аналитическое выражение для CWT $V(v, t)(6)$, использующее AWM (2). Зависимостью $|V(v, t)|$ от времени $t$ для такого сигнала можно пренебречь, так как эти слагаемые имеют малость $O\left(\exp \left(-2 \Omega_{m}^{2}\right)\right)$. Это преимущество комплексного AWM перед другими вещественными материнскими вейвлетами (DOG, MHAT), для которых $|V(v, t)|$ зависит от $t$. Максимум $|V(v, t)|$ расположен в точке $v=f_{0}$. Полная ширина $|V(v, t)|$ на половине максимума пика $2 \Delta_{\mathrm{FWHM}} \approx \sqrt{2 \ln 2} f_{0} /(\pi m)$ может быть уменьшена при увеличении параметра $m$. 
Для суммы двух гармонических сигналов бесконечной протяженности $Z(t)=\cos \left(2 \pi f_{K} t\right)+\cos \left(2 \pi f_{L} t\right)$ с различными частотами $f_{K}>f_{L}$ также можно получить аналитическое выражение для $V(v, t)(6)$. Поверхность $|V(v, t)|$ показывает существование двух „холмов“, вытянутых вдоль оси времени $t$. Если частоты $f_{K}$ и $f_{L}$ сильно отличаются друг от друга, то эти холмы, параллельные оси времени, сильно разнесены друг от друга по оси частот. Если частоты $f_{K}$ и $f_{L}$ близки, то соответствующие холмы начинают перекрываться. Введем среднюю частоту $f=\left(f_{K}+f_{L}\right) / 2$ и разностную частоту $\Delta f=f_{K}-f_{L}$. Критерием разрешимости двух пиков является условие возникновения положительности второй производной $d^{2}|V(v, t)| / d v^{2}>0$ при условии $f_{L}<v<f_{K}$. Условие разрешения двух бесконечных гармонических сигналов имеет вид $\Delta f \geq f /(\pi m)$, что дает выигрыш в $m$ раз для AWM (1), (2) по сравнению с условием для обычного вейвлета Морле $(m=1)[1,3,23,33]$.

\section{Математическая модель нестационарного сигнала}

Рассмотрим модель, в которой сигнал $Z(t)$ может быть представлен в виде суперпозиции $N$ ENS $z_{L}\left(t-t_{L}\right)$ :

$$
\begin{gathered}
Z(t)=\sum_{L=0}^{N-1} z_{L}\left(t-t_{L}\right) . \\
z_{L}\left(t-t_{L}\right)=\frac{b_{L}}{2 \tau_{L} \sqrt{\pi}} \exp \left(-\frac{\left(t-t_{L}\right)^{2}}{4 \tau_{L}^{2}}\right) \\
\\
\times \cos \left(2 \pi f_{L}\left(t-t_{L}\right)+\alpha_{L}\right) .
\end{gathered}
$$

Каждый ENS имеет свою частоту $f_{L}(\mathrm{~Hz})$ и фазу $\alpha_{L}$ (rad). Центр ENS находится по времени в точке $t_{L}(\mathrm{~s})$, имеет ширину локализации $\tau_{L}(\mathrm{~s})$ и характеризуется амплитудой $b_{L}$. Пять параметров каждого ENS $z_{L}\left(t-t_{L}\right)$ формируют одномерный массив $L=\left(b_{L}, f_{L}, t_{L}, \tau_{L}, \alpha_{L}\right)$. Принцип суперпозиции позволяет представить CWT $V(v, t)$ (6) суммарного нестационарного сигнала $Z(t)(12)$ в виде суммы CWT для каждого отдельного ENS $V_{L}(v, t)$. Величина $V_{L}(v, t)$, вычисленная для $\mathrm{ENS} z_{L}\left(t-t_{L}\right)$ с помощью AWM, имеет вид

$$
\begin{gathered}
V_{L}(v, t)=V_{L}^{(+)}\left(v, t, f_{L}, \alpha_{L}\right)+V_{L}^{(+)}\left(v, t,-f_{L},-\alpha_{L}\right), \\
V_{L}^{(+)}\left(v, t, f_{L}, \alpha_{L}\right)=\frac{m b_{L} D_{m} \exp \left[-\frac{\left(t-t_{L}\right)^{2}}{4 \tau_{L}^{2} \kappa_{L}^{2}}\right]}{\sqrt{8} \tau_{L} \kappa_{L}} \\
\times\left\{\exp \left[i \delta_{L}-\frac{\Omega_{m}^{2}\left(f_{L}-v\right)^{2}}{v^{2} \kappa_{L}^{2}}\right]-\exp \left[i \gamma_{L}-\Omega_{m}^{2}-\frac{\left.\Omega_{m}^{2} f_{L}^{2}\right]}{v^{2} \kappa_{L}^{2}}\right]\right\}, \\
\kappa_{L}^{2}=1+m^{2} /\left(2 v^{2} \tau_{L}^{2}\right), \gamma_{L}=\alpha_{L}+2 \pi f_{L}\left(t-t_{L}\right) / \kappa_{L}^{2},
\end{gathered}
$$

Анализируя выражение (14), получаем, что при рассмотрении положительных частот $v>0$ для CWT вкладом $V_{L}^{(+)}\left(\nu, t,-f_{L},-\alpha_{L}\right)(15)$ можно пренебречь, так как параметр $\Omega_{m}^{2}=2 \pi^{2} m^{2} \gg 1$. Обычно для ENS (13) выполняется условие $f_{L} \tau_{L} \gg 1$, которое означает, что на характерном интервале $\tau_{L}$ помещается много периодов гармонических колебаний $1 / f_{L}$. При условии $f_{L} \tau_{L} \gg 1$ для частот $v \approx f_{L}$, характеризующих максимальное значение $\left|V_{L}(v, t)\right|$, функции $\kappa_{L} \approx 1(16)$, если выполняется неравенство $m \ll f_{L} \tau_{L}$. В этом случае поведение амплитуды $\left|V_{L}(v, t)\right|$ со временем $t$ практически совпадает с поведением амплитуды сигнала ENS (13). Максимум $\left|V_{L}(v, t)\right|$ по частоте $v$ наблюдается при $v=f_{L}$, а по времени $t$ в момент времени $t_{L}$. С увеличением управляющего параметра $m$ область локализации сигнала по частотам может быть значительно уменьшена. Полная ширина пика $\left|V_{L}(v, t)\right|$, зависящего от частоты $v$, на половине своего максимума равняется $2 \Delta_{\mathrm{FWHM}} \approx \sqrt{2 \ln 2} f_{L} /(\pi m)$.

Анализ формул (14)-(17) для $\left|V_{L}(v, t)\right|$ показывает, что для ENS с параметрами $L=(3,2,12,3,0)$ изменение параметра $m$ приводит к незначительному изменению протяженности $\left|V_{L}(v, t)\right|$ по оси времени $t$. Так, при $v_{L}=2 \mathrm{~Hz}, \tau_{L}=3 \mathrm{~s}$ величина $\kappa_{L}$ изменяется с $\kappa_{L}=1.007$ при $m=1$, до $\kappa_{L}=1.16$ при $m=5$. Локализация $\left|V_{L}(v, t)\right|$ по оси частот $v$ при увеличении параметра $m$ меняется довольно значительно, так как ширина пика в точке $t=t_{L}$, равная $\Delta_{\mathrm{FWHM}}$, обратно пропорциональна величине $m$.

Продемонстрируем преимущества применения AWM для анализа реального сигнала $Z(t)$, представляющего собой сумму двух ENS (13) с параметрами $S=\left(b_{S}, f_{S}, t_{S}, \tau_{S}, \alpha_{S}\right)=(3,2,12,3,0), \quad W=$ $=\left(b_{W}, f_{W}, t_{W}, \tau_{W}, \alpha_{W}\right)=(1,3,12,1,0), \quad$ и случайного сигнала с амплитудой $A$

$$
Z(t)=z_{S}\left(t-t_{S}\right)+z_{W}\left(t-t_{W}\right)+A(\operatorname{Random}(t)-0.5),
$$

где $\operatorname{Random}(t)-$ случайная функция, изменяющаяся в интервале $0 \leq \operatorname{Random}(t) \leq 1$. Отметим, что два ENS с приведенными параметрами $S$ и $W$ имеют одинаковые центры локализации $t_{S}=t_{W}=12 \mathrm{~s}$, различные частоты

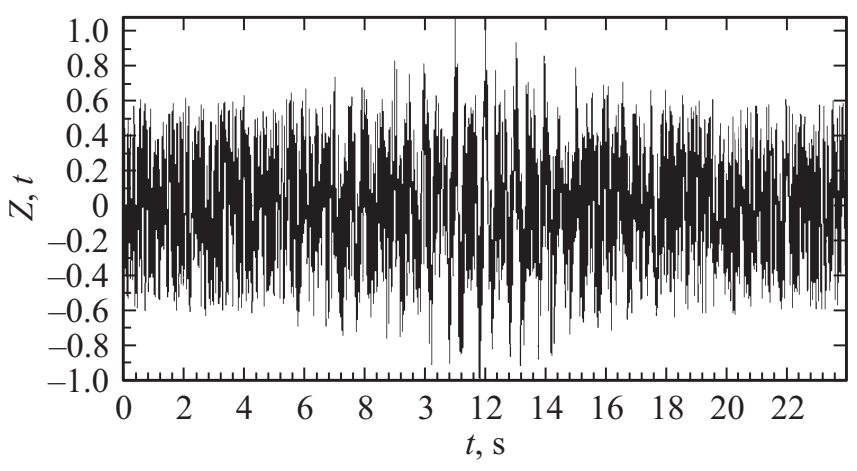

Рис. 1. Зависимость сигнала $Z(t)(18)$ от времени $t$, s. Параметры $S=(3,2,12,3,0), W=(1,3,12,1,0), A=1.2$. 


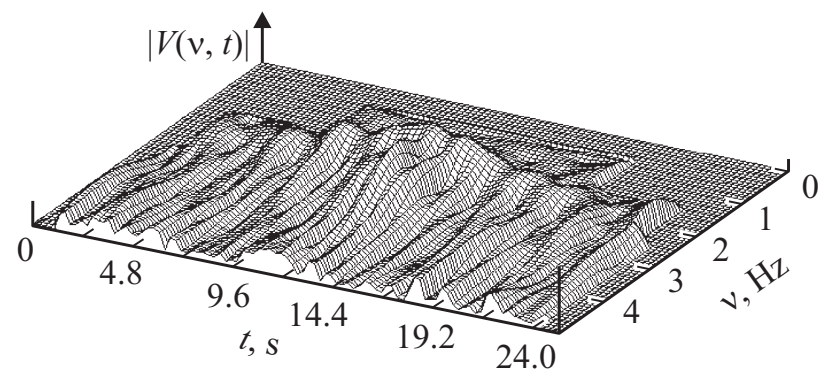

Рис. 2. График величины $|V(v, t)|(6)$ в зависимости от времени $t, \mathrm{~s}$ и частоты $v, \mathrm{~Hz}$ для сигнала $Z(t)(18)$ (рис. 1), построенный с помощью $\mathrm{AWM}(m=1)(1)$.

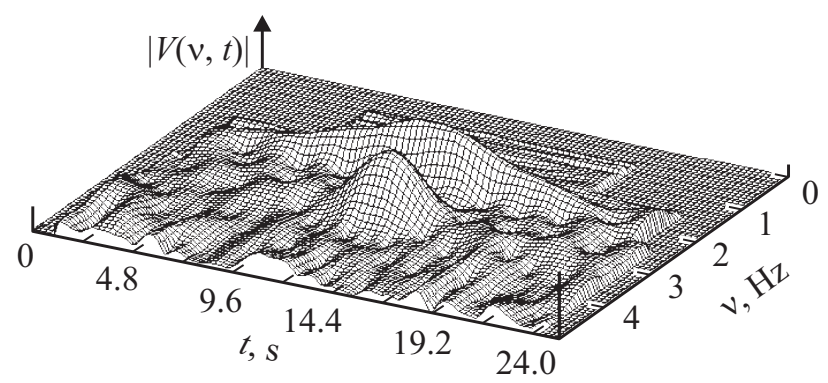

Рис. 3. График величины $|V(v, t)|(6)$ в зависимости от времени $t, \mathrm{~s}$ и частоты $v, \mathrm{~Hz}$ для сигнала $Z(t)(18)$ (рис. 1), построенный с помощью $\mathrm{AWM}(m=2)(1)$.

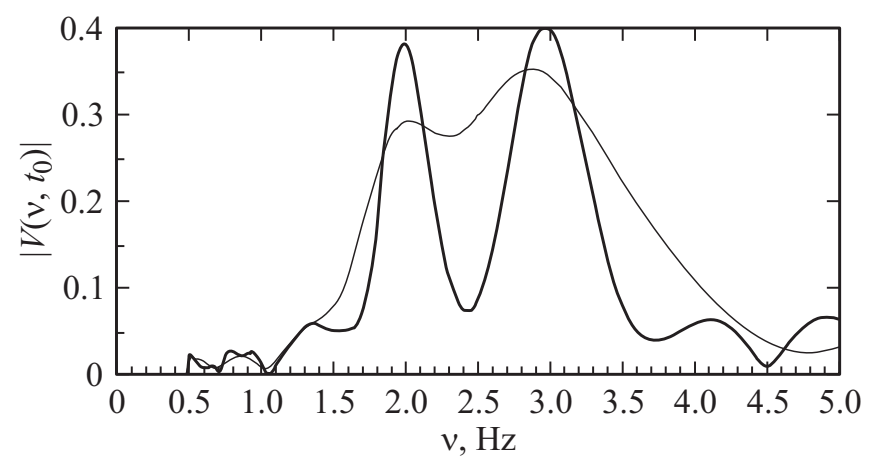

Рис. 4. Разрез CWT $\left|V\left(v, t_{0}\right)\right|$, выполненный в точке $t_{0}=12 \mathrm{~s}$, в зависимости от частоты $v, \mathrm{~Hz}$ для сигнала $Z(t)(18)$. Тонкой линией показан разрез для $m=1$, толстой линией $-m=2$.

$f_{S}=2 \mathrm{~Hz}, f_{W}=3 \mathrm{~Hz}$ и радиусы локализации $\tau_{S}=3 \mathrm{~s}$, $\tau_{W}=1 \mathrm{~s}$. На рис. 1 изображен сигнал $Z(t)$ (18) для случая $A=1.2$. При $A=1.2$ максимальные значения суммарного стохастического сигнала $Z(t)(18)$ примерно в 2 раза превосходят сумму двух $\mathrm{ENS} z_{S}\left(t-t_{S}\right)$ и $z_{W}\left(t-t_{W}\right)$ с теми же значениями параметров $S$ и $W$, вычисленных в момент времени $t_{0}=t_{S}=t_{W}$. На рис. 2,3 представлен результат численного расчета поверхности $|V(v, t)|(6)$, зависящей от частоты $v$ и времени $t$, для модельного сигнала $Z(t)(18)$, причем на рис. 2 управляющий параметр AWM $m=1$, а на рис. 3 $m=2$. Сравнение рис. 2 и 3 показывает, что при увеличении управляющего параметра $m$ в 2 раза (рис. 3 ) удается спектрально разрешить суперпозицию двух ENS, имеющих частоты $f_{S}$ и $f_{W}$, зашумленных случайным сигналом с амплитудой $A=1.2$. На рис. 4 изображен разрез поверхности $\left|V\left(v, t_{0}\right)\right|$ в зависимости от частоты $v$ в момент времени $t_{0}=12 \mathrm{~s}$. Тонкой линией обозначен разрез поверхности с $m=1$ (плохое спектральное разрешение двух пиков $f_{S}$ и $\left.f_{W}\right)$, толстой линией $-m=2$ (хорошее спектральное разрешение). Таким образом, увеличивая в 2 раза управляющий параметр AWM при вычислении $|V(v, t)|$, мы смогли спектрально разрешить два ENS, сильно зашумленных случайным сигналом.

\section{Корреляционная функция сигналов}

Для любых сигналов $Z_{\alpha}(t)$ и $Z_{\beta}(t)$ можно ввести кросскорреляционную функцию (CCF) [4,5]

$$
\mathrm{CCF}_{\alpha \beta}(t)=\int_{-\infty}^{\infty} Z_{\alpha}\left(t^{\prime}\right) Z_{\beta}\left(t+t^{\prime}\right) d t^{\prime}
$$

Функция $\mathrm{CCF}_{\alpha \beta}(t)$ характеризует взаимосвязь между сигналами, взятыми в различные моменты времени. Если в качестве сигнала $Z_{\alpha}(t)$ взять ENS $z_{L}(t)$ с параметрами $L$, а в качестве $Z_{\beta}(t)$ взять ENS $z_{K}(t)$ с параметрами $K$, то в этом случае $\mathrm{CCF}_{L K}(t)$ равна

$$
\begin{gathered}
\mathrm{CCF}_{L K}(t)=\frac{b_{L} b_{K} \exp \left(-\frac{t_{0}^{2}}{4 \tau_{\Sigma}^{2}}\right)}{4 \tau_{\Sigma} \sqrt{\pi}}\left\{\exp \left[-\left(F_{K 0}+F_{L 0}\right)^{2}\right] \cos \gamma_{+}\right. \\
\left.+\exp \left[-\left(F_{K 0}-F_{L 0}\right)^{2}\right] \cos \gamma_{-}\right\} \\
t_{0}=t-\left(t_{K}-t_{L}\right), \quad \tau_{\Sigma}^{2}=\tau_{K}^{2}+\tau_{L}^{2}, \tau_{0}=\tau_{K} \tau_{L} / \sqrt{\tau_{K}^{2}+\tau_{L}^{2}}, \\
F_{K 0}=2 \pi f_{K} \tau_{0}, \quad F_{L 0}=2 \pi f_{L} \tau_{0}, \\
\gamma_{+}=\frac{2 \pi\left[f_{K} \tau_{K}^{2}-f_{L} \tau_{L}^{2}\right] t_{0}}{\tau_{K}^{2}+\tau_{L}^{2}}+\alpha_{K}+\alpha_{L} \\
\gamma_{-}=\frac{2 \pi\left[f_{K} \tau_{K}^{2}+f_{L} \tau_{L}^{2}\right] t_{0}}{\tau_{K}^{2}+\tau_{L}^{2}}+\alpha_{K}-\alpha_{L}
\end{gathered}
$$

Анализируя соотношение (20), получаем, что при выполнении соотношений $f_{K} \tau_{0} \gg 1, f_{L} \tau_{0} \gg 1$ основной вклад в $\mathrm{CCF}_{L K}(t)$ дает второе выражение в фигурной скобке, пропорциональное $\cos \gamma_{-}$. Если частоты простейших нестационарных сигналов одинаковы $f_{K}=f_{L}$, то $\mathrm{CCF}_{L K}(t)$, центрированная по времени в точке $t=t_{K}-t_{L}$, будет осциллировать с частотой $f_{K}$. Если частоты сигналов $f_{K}$ и $f_{L}$ отличаются друг от друга, то $\mathrm{CCF}_{L K}(t)$ будет уменьшаться по амплитуде на множитель $\exp \left(-\left(F_{K 0}-F_{L 0}\right)^{2}\right)$, а характерная частота осцилляций функции $\mathrm{CCF}_{L K}(t)$

$$
f_{L K}=\left(f_{K} \tau_{K}^{2}+f_{L} \tau_{L}^{2}\right) /\left(\tau_{K}^{2}+\tau_{L}^{2}\right) .
$$




\section{Двойная вейвлетная корреляционная функция нестационарных сигналов}

Предположим, что у нас существуют два NS $Z_{\alpha}(t)$ и $Z_{\beta}(t)$. Сигналу $Z_{\alpha}(t)$ соответствует CWT (12), равное $V_{\alpha}(v, t)$, а сигналу $Z_{\beta}(t)$ соответственно $V_{\beta}(v, t)$. Введем $\operatorname{DWC}_{\alpha \beta}(v, t)$ - двойную вейвлетную корреляционную функцию (DWC - double wavelet correlation) этих NS согласно соотношению

$$
\operatorname{DWC}_{\alpha \beta}(v, t)=\int_{-\infty}^{\infty} d t^{\prime} \int_{-\infty}^{\infty} d v^{\prime} V_{\alpha}^{*}\left(v^{\prime}, t^{\prime}\right) V_{\beta}\left(v+v^{\prime} ; t+t^{\prime}\right) .
$$

Предположим, что сигнал $Z_{\alpha}(t)$ будет представлять собой суперпозицию $N_{\alpha} \operatorname{ENS}(12),(13)$, каждый из которых будет характеризоваться одномерным вектором $L_{\mu}$, состоящим из пяти параметров. Сигнал $Z_{\beta}(t)$ будет представлять собой суперпозицию $N_{\beta}$ ENS (12), (13), каждый из которых будет характеризоваться одномерным вектором $K_{\nu}$. В этом случае $\operatorname{DWC}_{\alpha \beta}(v, t)(25)$ может быть выражена через комбинацию $\operatorname{DWC}_{L K}(v, t)$ двух ENS

$$
\operatorname{DWC}_{L K}(v, t)=\int_{-\infty}^{\infty} d t^{\prime} \int_{-\infty}^{\infty} d v^{\prime} V_{L}^{*}\left(v^{\prime}, t^{\prime}\right) V_{K}\left(v+v^{\prime} ; t+t^{\prime}\right) .
$$

В этом выражении $V_{L}(v, t)$ и $V_{K}(v, t)(14)$ представляют собой CWT (6) двух ENS $z_{L}\left(t-t_{K}\right)$ с вектором параметров $L=\left(b_{L}, f_{L}, t_{L}, \tau_{L}, \alpha_{L}\right)$ и $z_{K}\left(t-t_{K}\right)$ с вектором $K=\left(b_{K}, f_{K}, t_{K}, \tau_{K}, \alpha_{K}\right)$. Предположим, что частота сигнала $z_{K}\left(t-t_{K}\right)$, равная $f_{K}$, больше, чем частота сигнала $z_{L}\left(t-t_{L}\right)$, равная $f_{L}\left(f_{K}>f_{L}\right)$. В этом случае в функции $\operatorname{DWC}_{L K}(v, t)$ нам достаточно интересоваться лишь положительными значениями частоты $v>0$. Заметим, что интеграл по времени $t^{\prime}$ в выражении (26) с помощью (14)-(17) может быть вычислен точно. Учитывая только положительно частотные части в выражениях $V_{L}(\nu, t)$ и $V_{K}(v, t)$, с большой точностью функцию $\operatorname{DWC}_{L K}(v, t)$ можно выразить через однократный интеграл $I_{L K}\left(v, f_{L}, f_{K}\right)$ по частоте $v^{\prime}$, значение которого находится численно

$$
\begin{aligned}
\operatorname{DWC}_{L K}(v, t)= & \frac{b_{L} b_{K} m^{2} D_{m}^{2} \sqrt{\pi}}{4 \tau_{\Sigma}} \exp \left[i \gamma_{L K}-\frac{t_{0}^{2}}{4 \tau_{\Sigma}^{2}}\right. \\
& \left.-4 \pi^{2}\left(f_{L}-f_{K}\right)^{2} \tau_{0}^{2}\right] I_{L K}\left(v, f_{L}, f_{K}\right) .
\end{aligned}
$$

В выражении (27)

$$
\begin{gathered}
t_{0}=t-\left(t_{K}-t_{L}\right), v_{0}=v-\left(f_{K}-f_{L}\right), \tau_{\Sigma}=\sqrt{\tau_{L}^{2}+\tau_{K}^{2}}, \\
\tau_{0}=\tau_{L} \tau_{K} / \tau_{\Sigma}, \quad \gamma_{L K}=\alpha_{K}-\alpha_{L}+2 \pi\left(f_{L} \tau_{L}^{2}+f_{K} \tau_{K}^{2}\right) / \tau_{\Sigma}^{2} .
\end{gathered}
$$

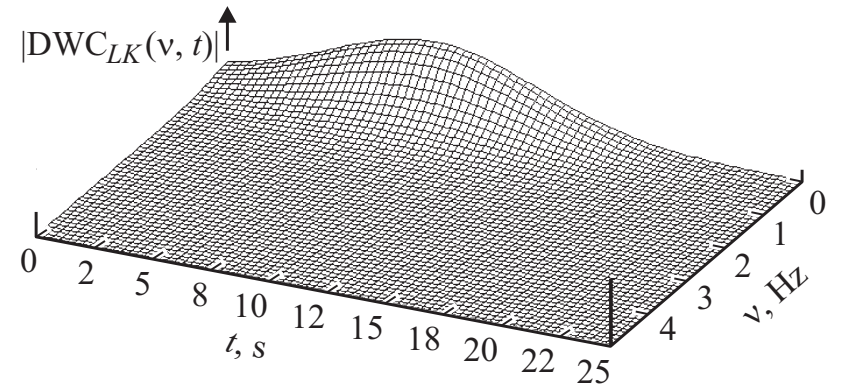

Рис. 5. Модуль двойной вейвлетной корреляционной функции $\left|\mathrm{DWC}_{L K}(v, t)\right|$ в зависимости от частоты $v, \mathrm{~Hz}$ и времени $t, \mathrm{~s}$ для двух ENS-сигналов $z_{L}\left(t-t_{L}\right)$ и $z_{K}\left(t-t_{K}\right)(13)$ с одинаковыми частотами, где $L=(3,2,12,3,0), K=(1,2,21,1,0)$, $m=1(1)$.

Поведение $\mathrm{DWC}_{L K}(v, t)$ по времени характеризуется гауссовой формой. Функция $\mathrm{DWC}_{L K}(v, t)$ имеет максимум по времени в точке $t_{0}=t-\left(t_{K}-t_{L}\right)=0$, и ее характерная ширина по времени определяется величиной $\tau_{\Sigma}(28)$. Если частоты элементарных нестационарных сигналов различны, то в этом случае $\mathrm{DWC}_{L K}(\nu, t)$ становится очень малой. Однократный интеграл $I_{L K}\left(v, f_{L}, f_{K}\right)$ в (27) имеет вид

$$
\begin{aligned}
& I_{L K}\left(v, f_{L}, f_{K}\right)=\exp \left(-2 \Omega_{m}^{2}\right) \int_{-\infty}^{\infty} \exp \left(-\frac{\Omega_{m}^{2} f_{L}^{2}}{\left(v^{\prime}+f_{L}\right)^{2}}\right) \\
& \quad \times\left[\exp \left(\frac{2 \Omega_{m}^{2} f_{L}}{v^{\prime}+f_{L}}\right)-1\right] \exp \left(-\frac{\Omega_{m}^{2} f_{K}^{2}}{\left(v^{\prime}+v_{0}+f_{K}\right)^{2}}\right) \\
& \quad \times\left[\exp \left(\frac{2 \Omega_{m}^{2} f_{K}}{v^{\prime}+v_{0}+f_{L}}\right)-1\right] d v^{\prime} .
\end{aligned}
$$

Анализ показывает, что максимальное значение $I_{L K}\left(v, f_{L}, f_{K}\right)$ достигается при частотах $v$, при которых $v_{0}=v-\left(f_{K}-f_{L}\right)=0$. На рис. 5 построена функция $\left|\mathrm{DWC}_{L K}(v, t)\right|$ для двух элементарных сигналов с одинаковыми частотами $f_{L}=f_{K}=2 \dot{\mathrm{Hz}}$, центрированных в различных точках по времени $t_{L}=$ $=12 \mathrm{~s}, t_{K}=21 \mathrm{~s}$. В этом случае по времени функция $\left|\operatorname{DWC}_{L K}(v, t)\right|$ имеет максимум в точке $t=9 \mathrm{~s}$ $\left(t_{0}=0(28)\right)$, а по частоте $v$ функция $\left|\operatorname{DWC}_{L K}(v, t)\right|$ имеет максимум в точке $v=0\left(v_{0}=0(28)\right)$. На рис. 6 для управляющего параметра $m=1$ построена функция $\left|\mathrm{DWC}_{L K}(v, t)\right|$ для двух ENS-сигналов с разными частотами $f_{L}=2, f_{L}=3 \mathrm{~Hz}$, центрированных в различных точках по времени $t_{L}=12 \mathrm{~s}, t_{K}=21 \mathrm{~s}$. В этом случае функция $\left|\mathrm{DWC}_{L K}(v, t)\right|$ по времени имеет максимум в точке $t=9 \mathrm{~s}\left(t_{0}=0(28)\right)$, а по частоте $v$ функция $\left|\mathrm{DWC}_{L K}(v, t)\right|$ имеет максимум в точке $v=1 \mathrm{~Hz}$ $\left(v_{0}=0(28)\right)$.

Характерная частотная локализация функции $\operatorname{DWC}_{\alpha \beta}(v, t)$ может быть изменена с помощью управляющего параметра $m$. На рис. 7 для управляющего параметра $m=2$ построена функция $\left|\mathrm{DWC}_{L K}(\nu, t)\right|$ для 


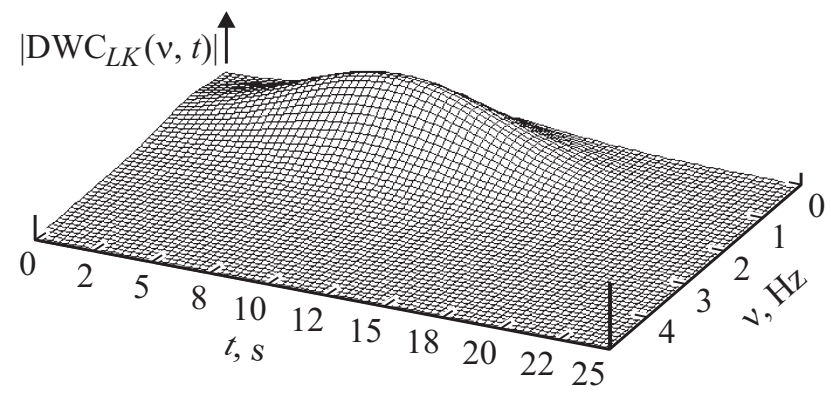

Pис. 6. Модуль двойной вейвлетной корреляционной функции $\left|\mathrm{DWC}_{L K}(v, t)\right|$ в зависимости от частоты $v, \mathrm{~Hz}$ и времени $t, \mathrm{~s}$ для двух ENS-сигналов $z_{L}\left(t-t_{L}\right)$ и $z_{K}\left(t-t_{K}\right)$ (13) с различными частотами, где $L=(3,2,12,3,0), K=(1,3,21,1,0)$, $m=1(1)$.

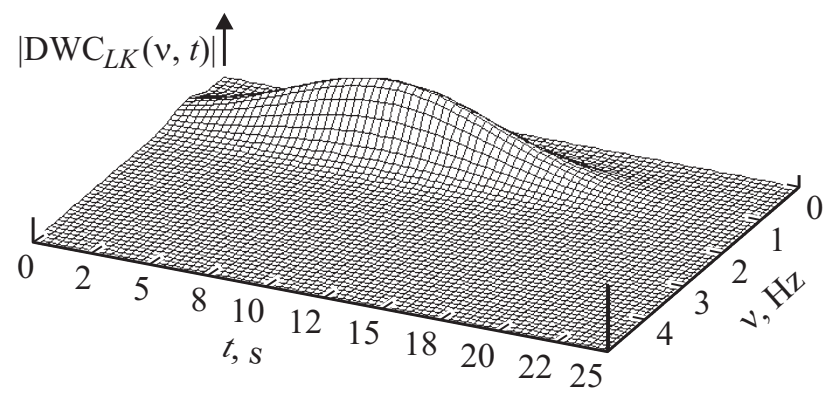

Рис. 7. Модуль двойной вейвлетной корреляционной функции $\left|\mathrm{DWC}_{L K}(v, t)\right|$ в зависимости от частоты $v, \mathrm{~Hz}$ и времени $t, \mathrm{~s}$ для двух ENS-сигналов $z_{L}\left(t-t_{L}\right)$ и $z_{K}\left(t-t_{K}\right)$ (13) с различными частотами, где $L=(3,2,12,3,0), K=(1,3,21,1,0)$, $m=2$.

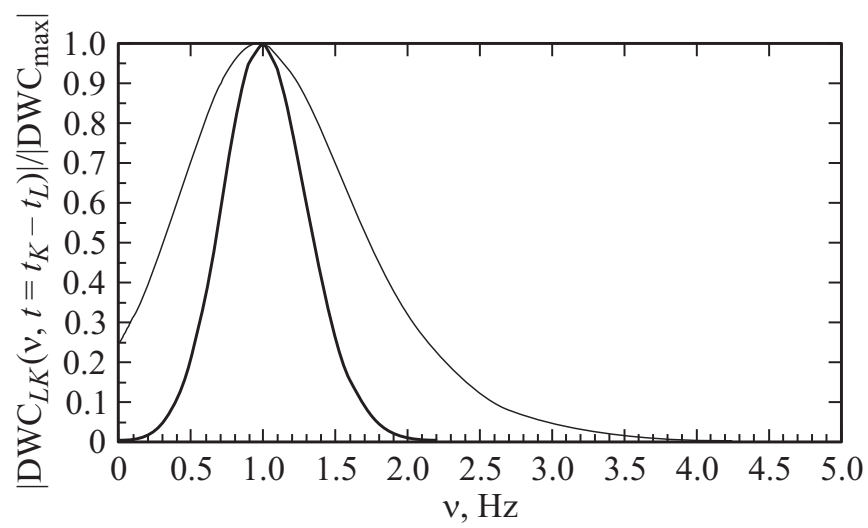

Рис. 8. Разрез величины $\frac{\left|\mathrm{DWC}_{L K}\left(v, t=t_{K}-t_{L}\right)\right|}{\left|\mathrm{DWC} C_{\max }\right|}$ в момент времени $t=t_{K}-t_{L} \quad\left(t_{0}=0 \mathrm{~s}(28)\right)$ в зависимости от частоты $v, \mathrm{~Hz}$. Сигналы $z_{L}\left(t-t_{L}\right)$ и $z_{K}=\left(t-t_{K}\right)$ характеризуются $L=(3,2,12,3,0), K=(1,3,21,1,0)(13)$. Тонкой линией построен разрез для $m=1$, толстой - для $m=2$.

двух элементарных сигналов с разными частотами $f_{L}=$ $=2, f_{K}=3 \mathrm{~Hz}$, центрированных в различных точках по времени $t_{L}=12 \mathrm{~s}, t_{K}=21 \mathrm{~s}$. Сравнивая поверхности, представленные на рис. 6 и 7 для аналогичных параметров двух ENS-сигналов, можно сделать вывод о том, что ширина частотной локализации $\operatorname{DWC}_{\alpha \beta}(v, t)$ для $m=2$ значительно меньше (рис. 7), чем $\mathrm{DWC}_{\alpha \beta}(v, t)$, построенная для $m=1$ (рис. 6). Разрез этих поверхностей $\mathrm{DWC}_{L K}(v, t)$ построен на рис. 8 при $t=9 \mathrm{~s}\left(t_{0}=0(35)\right)$ для двух ENS с параметрами $L=$ $=(3,2,12,3,0)$ и $K=(1,3,21,1,0)$. Тонкой линией показан разрез $\mathrm{DWC}_{L K}\left(v, t_{0}\right)$, выполненный с управляющим параметром $m=1$, толстой линией разрез с $m=2$.

\section{Заключение}

В настоящей работе проанализирована новая процедура исследования NS, которая основана на вычислении $V(v, t)(6)$ - CWT с помощью AWM (1), (2), зависящего от управляющего параметра $m$. Показано, что преимущество использования AWM заключается в возможности более точной диагностики частотной и временной локализаций исследуемого NS при помощи изменения управляющего параметра $m$ при вычислении $V(v, t)$. Получены критерии разрешимости спектральных пиков для двух ENS. Показано, что при увеличении $m$ степень разрешения может быть значительно улучшена.

Введена модель сложного сигнала $Z(t)(12)$ как суперпозиции ENS $z_{L}\left(t-t_{L}\right)(13)$. Каждый ENS представляет собой произведение огибающей гауссовой формы на осциллирующую функцию с фиксированной частотой. Получено аналитическое выражение CWT $V_{L}(v, t)(14)$ для ENS (13). Показано, что при увеличении управляющего параметра $m$ зависимость $V_{L}(v, t)$ от времени слабо меняется и в основном повторяет зависимость от времени сигнала ENS. Частотная локализация $V_{L}(v, t)$ при увеличении $m$ для ENS может быть значительно улучшена. Рассмотрена модель реального сигнала $Z(t)$, представляющего собой суперпозицию двух ENS и случайного сигнала. Показано, что, увеличивая параметр $m$, можно для CWT такого сигнала $V(v, t)$ выполнить спектральное разрешение двух ENS, имеющих близкие частоты.

Введена новая двойная вейвлетная корреляционная функция $\mathrm{DWC}_{\alpha \beta}(v, t)$, которая представляет собой CWT корреляцию величин $V_{\alpha}(v, t)$ и $V_{\beta}(v, t)$, вычисленных как по частоте $v$, так и по времени $t$ для двух нестационарных сигналов $Z_{\alpha}(t)$ и $Z_{\beta}(t)$. На примере двух ENS вычислено значение $\mathrm{DWC}_{L K}(v, t)$. Показано, что анализ поведения $\mathrm{DWC}_{L K}(v, t)$ по времени может определить разность времен локализации $t_{L}$ и $t_{K} \mathrm{ENS}$. Локализация $\mathrm{DWC}_{L K}(v, t)$ по частоте позволяет определить разность частот $f_{K}$ и $f_{L}$ двух ENS. Это является преимуществом $\operatorname{DWC}_{L K}(v, t)$ перед классической $\mathrm{CCF}_{L K}(t)$, которая не позволяет определять разность частот двух исследуемых сигналов. Показано, как с помощью параметра $m$ можно изменять частотное разрешение $\mathrm{DWC}_{L K}(v, t)$.

Предлагаемые подходы могут быть применены для анализа реальных нестационарных сигналов в физике, 
имеющих сложную динамику изменения как амплитудных, так и спектральных свойств. Увеличивая управляющий параметр $m$, можно улучшать условие разрешения спектральных свойств сигналов, имеющих близкие частоты.

В настоящее время большое значение имеет изучение электроэнцефалограммы (EEG), представляющей собой электрическую активность огромного количества нейронов, расположенных вблизи регистрирующего электрода $[27,28]$. Использование функции $\operatorname{DWC}_{\alpha \beta}(v, t)$ упростит описание синхронности и когерентности бегущих волн сигналов EEG, распространяющихся по коре головного мозга, и позволит вычислить динамику их корреляций [40]. Предлагаемый метод может быть применен для анализа корреляции вспышек EEG [16], а также вызванных потенциалов (ЕР - evoked potentials) кратковременных изменений электрической активности мозга, возникающих в ответ на сенсорную стимуляцию [41,42]. Применение $\mathrm{DWC}_{\alpha \beta}(v, t)$ к $\mathrm{EP}$ позволит установить количественные параметры, описывающие синхронность потенциалов ЕР в различных точках коры головного мозга. Использование $\mathrm{DWC}_{\alpha \beta}(v, t)$ может оказаться полезным при изучении быстроизменяющихся вспышечных процессов в физике плазмы и астрофизике $[32,43,44]$. Эта методика может быть применена для анализа корреляции вспышек на Солнце и исследовании сигналов пульсаров. Предлагаемый метод может найти свое применение для описания неравновесных явлений в теории взаимодействующих когерентных пространственно-временных структур, для описания сейсмологических сигналов $[45,46]$. Особенно важно применение $\mathrm{DWC}_{\alpha \beta}(v, t)$ для сред, обладающих сильной дисперсией. В этом случае необходимо находить корреляции между вспышками, частотный состав которых в процессе их распространения может претерпевать значительные изменения.

Известно, что для анализа стабильности работы квантового стандарта частоты (QSF) используется критерий, основанный на вычислении дисперсии Аллена [30,31]. Формула для дисперсии Аллена выведена в предположении нормального, стационарного, эргодического случайного процесса, когда флуктуации сигнала во временном представлении не коррелированы между собой. Однако для реальной ситуации в течение всего срока службы работы QSF могут развиваться различные переходные процессы [47-49]. В этом случае шумы QSF могут возникать и исчезать, а их спектральные характеристики будут изменяться во времени. Следовательно, такой процесс нельзя рассматривать как стационарный. Обобщение формулы Аллена требует разработки новых критериев для анализа нестабильности QSF. Для решения такой задачи вместо преобразования Фурье можно будет использовать CWT $V(v, t)$, а также предложенную в настоящей работе функцию $\mathrm{DWC}_{\alpha \beta}(v, t)$, опирающуюся на AWM.

Еще одним применением разрабатываемой в настоящей работе методики может стать изучение корре- ляционных свойств сигналов, получаемых с помощью фемтосекундных лазеров [50,51]. Нестационарность интерференционных процессов для ультракоротких источников излучения, имеющих различные пространственночастотные характеристики с сильно изменяющейся амплитудой во времени, требует новых подходов к расчету корреляции таких сигналов. В этом случае применение функции $\mathrm{DWC}_{\alpha \beta}(v, t)$ также поможет в интерпретации экспериментов с ультракороткими импульсами, имеющими сложный частотный состав.

Работа выполнена при финансовой поддержке Государственного задания № 3.1446.2014/К и ФЦП „Исследования и разработки по приоритетным направлениям научно-технологического комплекса России на 2014-2020 гг.“" (соглашение № 14.578.21.0211).

\section{Список литературы}

[1] Mallat S. A Wavelet Tour of Signal Processing, 3rd ed. NY.: Academic Press, 2008. 671 p.

[2] Chui C.K. An Introduction to Wavelets, NY.: Academic Press, 1992. $413 \mathrm{p}$.

[3] Daubechies I. Ten Lectures on Wavelet. Society for industrial and applied mathematics. Philadelphia, 1992. 463 p.

[4] Mandel L., Wolf E. Optical Coherence and Quantum Optics. Cambridge University Press, 1995. 1160 p.

[5] Yarlagadda R.K.R. Analog and Digital Signals and Systems. Springer, 2010. $488 \mathrm{p}$.

[6] Klein A., Sauer T., Jedynak A., Skrandies W. // IEEE Trans. Biomed. Eng. 2006. Vol. 53. N 2. P. 266-272.

[7] Pereda E., Quiroga R.Q., Bhattacharya J. // Progress in Neurobiology. 2005. Vol. 77. P. 1-37.

[8] Kulaichev A.P. // Neuroscience and Behavioral Physiology. 2001. Vol. 41. N 3. P. 321-328.

[9] Timashev F., Panischev O.Yu., Polyakov Y.S., Demin S.A., Kaplan A.Ya. // Physica A. 2012. Vol. 391. N 4. P. 1179 1194.

[10] Schönwald S.V., Gerhardt G.J.L., de Santa-Helena E.L., Chaves M.L.F. // Physica A. 2003. Vol. 327. N 1-2. P. 180 184.

[11] Paluš M., Dvořak I., David I. // Physica A. 1992. Vol. 185. N 1-4. P. 433-438.

[12] Rosso O.A., Martin M.T., Plastino A. // Physica A. 2005. Vol. 347. P. 444-464.

[13] Pereyra M.E., Lamberti P.W., Rosso O.A. // Physica A. 2007. Vol. 379. N 1. P. 122-132.

[14] Stratimirović D., Milošević S., Blesić S., Ljubisavljević M. // Physica A. 2007. Vol. 374. N 2. P. 699-706.

[15] Addison P.S. // Physiol. Meas. 2005. Vol. 26. R155-R199.

[16] Bozhokin S.V., Suslova I.B. // Physica A. 2015. Vol. 421. P. 151-160.

[17] Андреев А.А., Божсокин С.В., Веневцев И.Д., Жунусов К.Т. // ЖТФ. 2014. Т. 84. N 10. С. 10-15.

[18] Torrence C., Compo G.P. // Bull. Am. Meteorol. Soc. 1998. Vol. 79. P. 61-78.

[19] Lachaux J.-P., Lutz A., Rudrauf D., Cosmelli D., Quyen L.V., Martinerie J., Varela F. // Neurophysiologie Clinique. 2002. Vol. 32. P. 157-174.

[20] Grinsted A., Moore J.C., Jevrejeva S. // Nonlinear Processes in Geophys. 2004. Vol. 11. P. 561-566. 
[21] Li X., Yau X., Fox J., Jefferys J.G. // J. Neuroscience Methods. 2007. Vol. 160. P. 178-185.

[22] Qassim W.T., Cutmore T.R.H, James D.A., Rowland D.D. // Computers in Biology and Medicine. 2013. Vol. 43. P. 23-31.

[23] Hramov A.E., Koronovckii A.A., Makarov V.A., Pavlov A.N., Sitnikova E. Wavelets in neuroscience. Springer Series in Synergetics. Berlin, Heidelberg. Springer-Verlag, 2015. 331 p.

[24] Mizuno-Matsumoto Y., Ukai S., Isii R., Date S., Kaishima T., Shinosaki K., Shomojo S., Takeda M., Tamura S., Inouye T. // Brain Topography. 2005. Vol. 17. N 4. P. 237-252.

[25] Абарбанель Г.Д., Рабинович М.И., Селверстон А., Баженов М.В., Хуэрта Р., Сущик М.М., Рубчинский Л.Л. // УФН. 1996. Т. 166. N 4. С. 363-390.

[26] Chizhov A.V., Graham L.J. // Phys. Rev. E. 2008. Vol. 77. P. 011910-011917.

[27] Nunez P.L., Srinivasan R. // Electric Fields of the Brain: The Neurophysics of EEG. Second Edition. Oxford University Press, 2006.

[28] Herrmann C.S., Grigutsch M., Busch N.A. EEG Oscillations and Wavelet Analysis. Event-related Potentials: A methods handbook, Cambridge, MA: MIT Press, 2005.

[29] Zhdanova I.V., Masuda K., Bozhokin S.V., Rosene D.L., González-Martínez J., Schettler S., Samorodinsky E. // PLoS ONE. 2012. Vol. 7. N 3. P. E33327.

[30] Чирков А.Г., Матисов Б.Г. Современная теории стабильности прецизионных генераторов. СПб.: Изд-во Политех. ун-та., 2010. $214 \mathrm{c}$.

[31] Riley W.J. Handbook of Frequency Stability Analysis, National Institute of Standards and Technology. Washington, 2008.

[32] Ryabinkov A.I., Kaurov A.A., Kaminker A.D. // Astrophys. Space. Sci. 2013. Vol. 344. P. 219-228.

[33] Chui C.K., Jiang O. Applied Mathematics. Data Compression, Spectral Methods, Fourier Analysis, Wavelets and Applications. Mathematics Textbooks for Science and Engineering. Vol. 2. Atlantis Press, 2013.

[34] Павлов А.Н., Храмов А.Е., Короновский А.А., Ситникова Е.Ю., Макаров В.А, Овчинников А.А. // УФН. 2012. T. 182. N 9. C. $905-939$.

[35] Божскин С.В., Чобан Э.А. // ЖТФ. 1984. Т. 54. Вып. 10. C. $1865-1867$.

[36] Божскин С.В., Лыков С.Н. Дополнительные главы теоретической физики. Вейвлеты. Учебное пособие. Изд-во СПбГПУ, 2007. С. 252.

[37] Божскин С.В., Суворов Н.Б. // Биомедицинская радиоэлектроника. 2008. Вып. 3. С. 21-25.

[38] Божокин С.В. // ЖТФ. 2010. Т. 80. Вып. 9. С. 16-24.

[39] Божскин С.В. // ЖТФ. 2012. Т. 82. Вып. 7. С. 8-13.

[40] Трофимов А.Г., Колодкин И.В., Ушаков В.Л., Величковский Б.М. Сб. науч. тр. XVII Всерос. Научно-технической конф. Нейроинформатика-2015, М.: НИЯУ МИФИ, 2015. Ч. 1. С. $66-77$.

[41] Borodina U.V., Aliev R.R. // Neurocomputing. 2013. Vol. 121. P. 551-556.

[42] Romanov A.S., Sharova E.V., Kuznetsova O.A., Oknina L.B., Volynskii P.E., Shchekutiev G.A. // Neuroscience and Behavioral Physiology. 2012. Vol. 42. N 6. P. 588-593.

[43] Ellis J., Mavromatos N.E., Nanopoulos D.V., Sakharov A.S. // Astronomy and Astrophysics. 2003. Vol. 402. N 2. P. 409-424.

[44] Mészárosová H., Karlický M., Rybák J., Jiřička K. // Astroph. J. Lett. 2009. Vol. 697. L108-L110.

[45] Sinha S., Routh P.S., Anno P.D., Castagna J.P. // Geophysics. 2005. Vol. 70. N 6. P. 19-25.
[46] Kulesh M., Holschneider M., Diallo M.S., Xie Q., Scherbaum F. // Pure Appl. Geophys. 2005. Vol. 162. N 5. P. 843-855.

[47] Vanier J. // Appl. Phys. B. 2005. Vol. 81. N 4. P. 421-442.

[48] Зибров С.А., Величанский В.Л., Зибров А.С., Тайченачев А.В., Юдин В.И. // Письма в ЖЭТФ. 2005. Т. 82. N 8. C. 534-538.

[49] Kazakov G., Matisov B., Litvinov A., Mazets I. // J. Phys. B. 2007. Vol. 40. N 19. P. 3851-3860.

[50] Deng Y., Wang C., Chai L., Zhang Z. // Appl. Phys. B. 2005. Vol. 81. P. 1107-1111.

[51] Deng Y., Wu Z., Chai L., Wang C., Yamane K., Morita R., Yamashita M., Zhan Z. // Opt. Expres. 2005. Vol. 13. N 6. P. 2120-2126. 